\title{
Quality of life in children following mitral valve replacement
}

\author{
C van Doorn, R Yates, A Tunstill, M Elliott
}

\begin{abstract}
Objective-To measure the health related quality of life (QoL) following mitral valve replacement in childhood.

Design-Cross sectional study.

Setting-Tertiary referral centre.

Methods-19 patients, median age (range) 14.4 (9.7-25.4) years, were studied at a median of $7.6(0.5-11.2)$ years after their most recent mitral valve replacement. General health status was measured using age specific validated questionnaires. Ten children aged between 9-15 years completed the child health related quality of life questionnaire, and for nine older patients the UK version of the short form 36 was used. Specific questions were added to the existing questionnaires to study the effect of long term anticoagulation treatment.

Results-All patients in the younger age group reported impaired QoL. Five rated their QoL within the range of children with chronic physical disabilities, and in the remaining five it was worse. In the older age group, all but two patients perceived their QoL as normal or near normal compared with a reference population matched for sex and age. Having regular blood tests had a negative effect on QoL in three young children, and one older patient reported impaired QoL related to taking daily warfarin tablets.

Conclusions-In this small group, the effect of mitral valve replacement on QoL appears to be age specific, with more impairment in younger children. Long term anticoagulation treatment is well tolerated in most patients.

(Heart 2000;84:643-647)
\end{abstract}

Keywords: prosthetic mitral valve; heart valve replacement; child; quality of life

The placement of a prosthetic mitral valve in a child is occasionally necessary if the failing native valve cannot be repaired. ${ }^{1-3}$ Traditionally, the success of treatment has been measured in terms of survival, but with many children now expected to live long term after mitral valve replacement ${ }^{45}$ this has become a less powerful end point and it is increasingly important to gain insight into their quality of life (QoL). This is of particular importance after mitral valve replacement as the currently favoured mechanical prostheses require permanent anticoagulation, and this has obvious implications for the lifestyle of the child. We therefore studied the general health status and the effect of anticoagulation treatment on QoL in children after mitral valve replacement.

This study was part of a follow up study on all patients who underwent mitral valve replacement at Great Ormond Street Hospital for Children, London, UK, between January 1987 and December 1997. The characteristics of this patient population, mortality, morbidity, and haemodynamic status up to 11 years of age are detailed in a companion paper. ${ }^{6}$ This project was approved by the medical ethics committee of the Institute of Child Health and Great Ormond Street Hospital for Children.

\section{Methods}

STUDY POPULATION

Fifty four consecutive patients underwent mitral valve replacement at our institution between January 1987 and December 1997. All received a mechanical prosthesis. The 30 operative survivors who are currently resident in the UK were invited to attend the outpatient department for clinical evaluation and echocardiographic examination. The results of these studies are reported elsewhere. ${ }^{6}$ In addition, patients underwent QoL assessment. These studies form the basis of this report.

Six patients were too young to complete a QoL questionnaire. Of the remaining 24 patients, five were excluded for the following reasons: no response to invitation, refusal to participate, end stage heart failure with palliative care at home, end stage heart failure awaiting heart transplant, and progressive loss of vision with profound effect on QoL. Two patients who declined to come to the outpatient clinic agreed to complete the questionnaire by mail and were included in the study. Nineteen patients, median (range) age 14.5 (9.7-25.4) years, were studied $7.6(0.5-11.2)$ years after their most recent mitral valve replacement. Twelve were female and seven male. Cardiac diagnoses leading to mitral valve replacement are listed in table 1 .

\section{QUALITY OF LIFE STUDIES}

There is no universally accepted definition of QoL or standard tool to measure it. We used age specific validated questionnaires to measure the patient's current general health status. To investigate the effect of anticoagulation treatment on QoL, special questions were added about taking daily warfarin tablets and having regular blood tests. The self administered questionnaires took approximately 15 
Table 1 Cardiac diagnoses in patients that underwent mitral valve replacement

\begin{tabular}{ll}
\hline Diagnosis & $\begin{array}{l}\text { Number of } \\
\text { patients }\end{array}$ \\
\hline Congenital mitral regurgitation & 5 \\
Congenital mitral stenosis & 4 \\
Rheumatic fever & 2 \\
Atrioventricular septal defect & 1 \\
Atrioventricular septal defect + tetralogy of & 1 \\
$\quad$ Fallot & 2 \\
Partial atrioventricular septal defect & 4 \\
Prosthetic mitral valve stenosis (original & \\
$\quad$ diagnosis: congenital mitral stenosis 3, viral & \\
$\quad$ mitral regurgitation 1) & 19 \\
Total & \\
\hline
\end{tabular}

minutes to complete. One of the investigators (AT) was available on site if assistance was required. Patients were studied in two age groups: 9-15 years, and 16 years and older.

Patients between 9-15 years old

Children aged 9-15 years were asked to complete the child health related quality of life (CQOL) questionnaire, which has 15 questions covering the child's functioning in areas of its daily life that are commonly affected by health problems. ${ }^{7}$ These domains were: getting about and using hands, doing things for self, soiling or wetting, school, out of school activities, friends, family relationships, discomfort caused by bodily symptoms, worries, depression, seeing, communication, eating, sleep, and appearance. An almost identical version of the questionnaire was completed by the parents, thus obtaining both the child's and the parents' view about the child's current functioning. To investigate the effect of permanent anticoagulant treatment on QoL, questions about the child's level of function, upset, and satisfaction in relation to taking daily warfarin tablets and having regular blood tests were added in the same format as the CQOL questionnaire (fig 1).

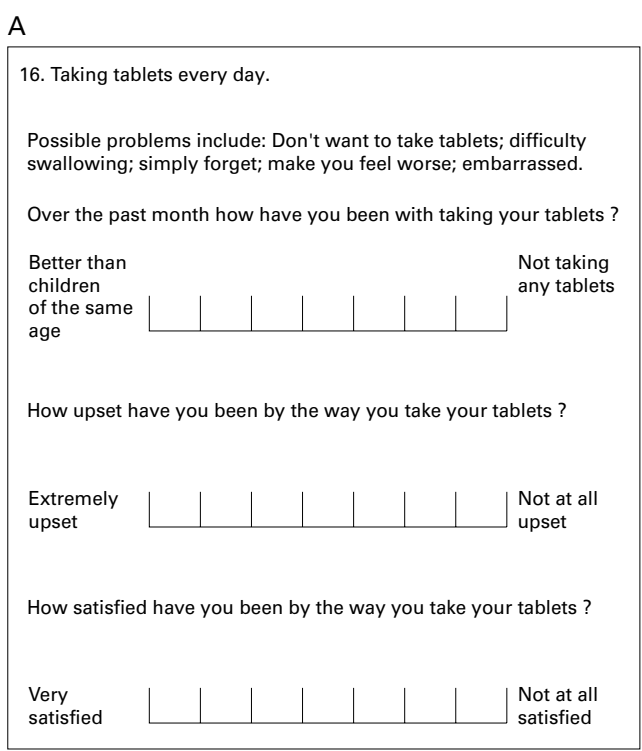

Patients aged 16 years and over

The general health status for patients aged 16 years and above was evaluated using the UK version of the short form 36 health survey (SF36). ${ }^{8}$ This self completion questionnaire contains 10 sections with a total of 36 questions, examining the following eight dimensions of health: physical functioning, social functioning, role limitation because of physical problems, role limitation because of emotional problems, mental health, energy/vitality, pain, and general health perception. To examine the effect of long term anticoagulation treatment, further questions were added to the SF-36 (fig 2).

\section{DATA HANDLING}

The QoL questionnaires were scored as published in the accompanying manuals and literature (see below). ${ }^{8-10}$ A meaningful statistical summary of the results was not possible because the patient population was small and heterogeneous. Instead the score of an individual patient was compared with that of published reference groups. Normal score was arbitrarily defined as within one standard deviation of the mean of the reference group. No reference values were available for the questions on anticoagulant treatment.

For the CQOL questionnaire the children and their parents marked the functional performance of the child on a visual seven pointed scale ranging from "better than children of the same age" (one point) to "very poor performance" (seven points). A cumulative CQOL score for each child was calculated by summation of the scores for all 15 questions, lower scores indicating better QoL. This score was compared with that of a published reference group of school children and a group with chronic physical disorders, including cystic fibrosis and orthopaedic and neurological disorders. ${ }^{9}$ Questions about anticoagulation

B

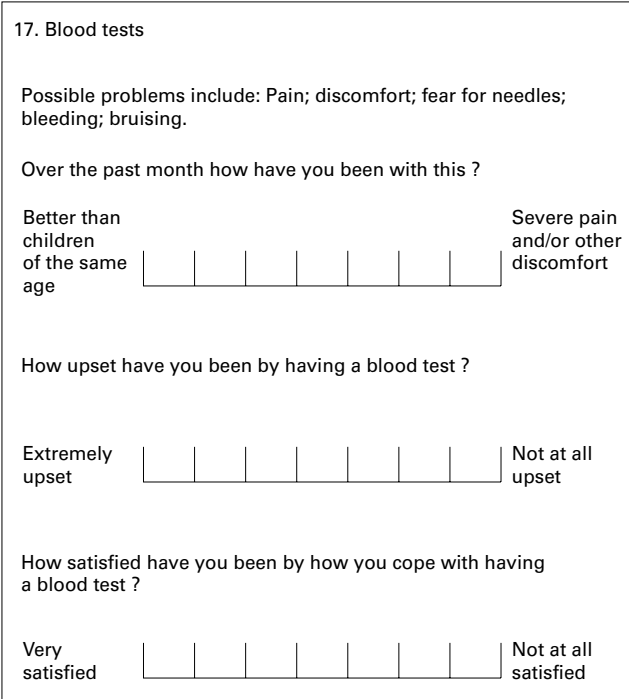

Figure 1 Questions added to the CQOL questionnaire to investigate the effect of taking daily tablets ( $A$ ) and having regular blood tests (B). Scoring of the level of function, upset, and satisfaction was on a seven point scale, ranging from "better than children of the same age" (1 point) to "not taking any tablets" (7 points); "not at all upset" (1 point) to "extremely upset" (7 points); and "very satisfied" (1 point) to "not at all satisfied" (7 points). 


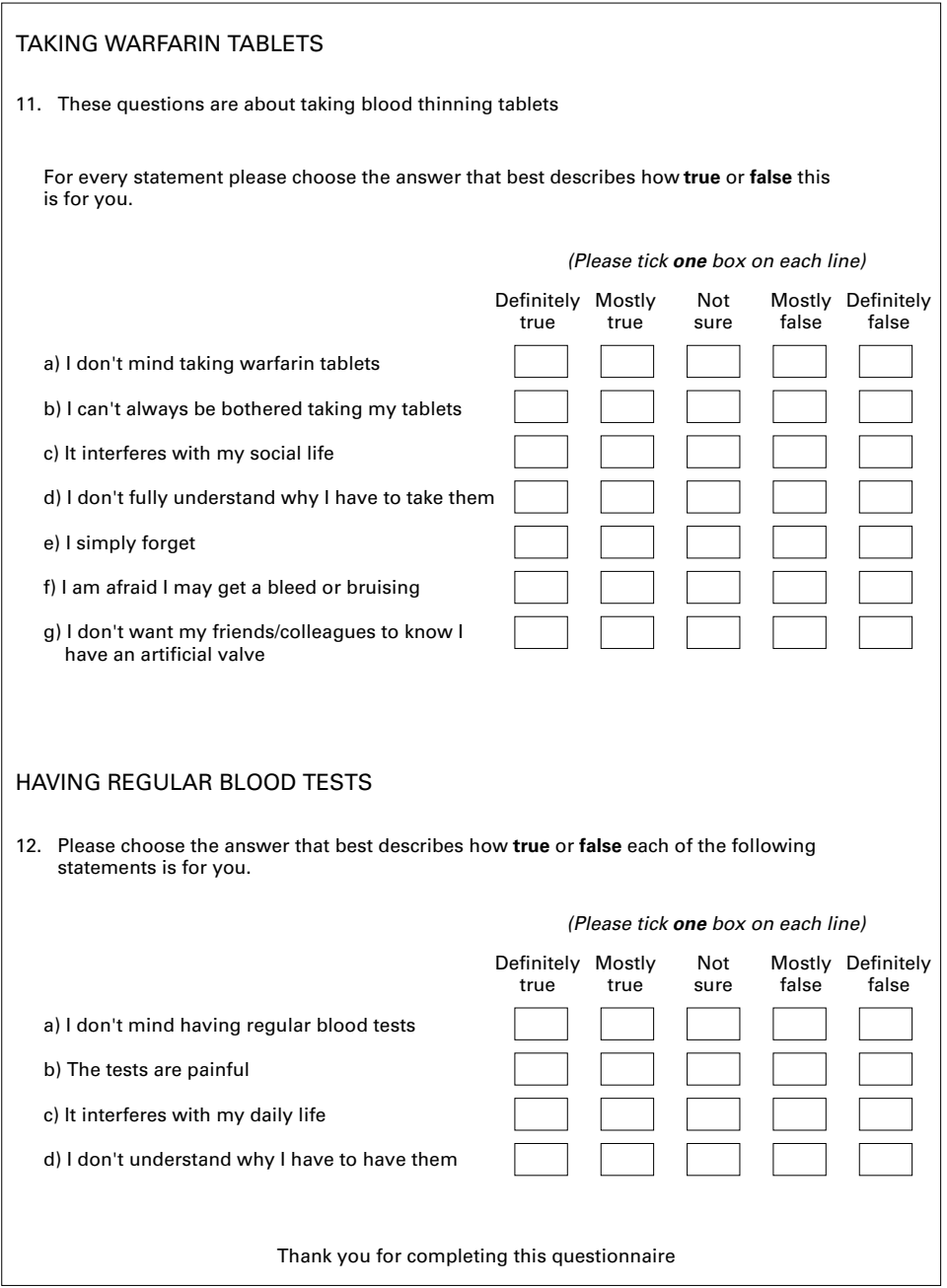

Figure 2 Questions added to the SF-36 questionnaire to measure the effect of permanent anticoagulation on quality of life. Scoring was on a five point scale. Questions 11a and 12 a: "definitely false" (1 point) to "definitely true" (5 points); the remaining questions were scored in reverse order: "definitely true" (1 point) to "definitely false" (5 points). The cumulative score for question 11 was calculated as: $(a+b+c+d+e+f+g-7) / 28 \times 100$, and for question 12 as: $(a+b+c+d-4) / 16 \times 100$. The cumulative score ranged from worst effect (0 points) to no adverse effect (100 points) on quality of life.

treatment were rated according to the same scoring system.

For each health dimension of the SF-36, the scores to the questions were coded according to the manual, summed, and transformed on to a scale from 0 (worst health) to 100 (best health). The total score for an individual patient was compared with published reference groups matched for sex and age. ${ }^{8}$ The effect of permanent anticoagulation on QoL was scored in a similar way and ranged from 0 (worst effect) to 100 (no adverse effect) (fig 2).

\section{Results}

PATIENTS BETWEEN 9-15 YEARS OLD

Ten patients (five girls, five boys), 12.4 (9.7-14.5) years old, were studied $6.2(0.5-$ 9.3) years after their most recent mitral valve replacement. All children attended school; nine were in mainstream education (one patient received additional help) and one at a special needs school because of delayed development.

The cumulative CQOL score for the children's perception of their physical functioning ranged from 21-69 and was outside the

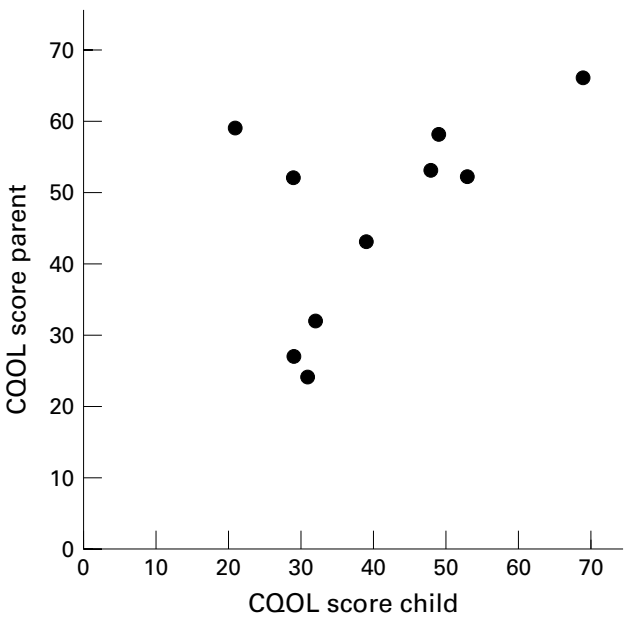

Figure 3 Perception of quality of life, child versus parent. In most cases the scores of the child and the parent were in good agreement. Two children scored much lower than their parents. CQOL, child health related quality of life. Lower scores indicate better quality of life.

reference range for normal children (14.9$19.6)^{9}$ in all cases. In five children it was within the range of children with chronic physical disabilities (15.3-35.7), ${ }^{9}$ and the remaining five children rated their physical functioning inferior to this group.

Figure 3 shows the relation between the CQOL scores of children and their parents. In most cases there was agreement between the children's and the parents' perception of the child's functioning. In two cases, however, children rated their functioning much better than their parents did.

The effects on QoL of taking daily warfarin tablets and having regular blood tests are summarised in fig 4 . All children reported that they were good at taking their daily tablets, not upset by taking them, and satisfied with their performance in this area. Almost identical ratings for the child's QoL were obtained from their parents. Having regular blood tests was associated with a good QoL in the majority of patients. In two patients QoL was moderately impaired. In one patient, who suffered from needle phobia and delayed development, it was associated with the worst QoL he could imagine. The parent scores were generally in agreement, except in two cases where parents rated their child's QoL much better and much worse, respectively.

PATIENTS AGED 16 YEARS AND OVER

Nine patients (seven female, two male), 19.9 (16.9-25.4) years old, were studied 7.6 (2.511.2) years after their last mitral valve replacement. Two patients were known to suffer from other health problems - moderate aortic regurgitation and asthma, respectively. All except one were in full time employment (5) or education (3). One patient, who was in good health, had elected to work part time.

Table 2 shows the SF-36 scores of individual patients compared with a reference population matched for sex and age. ${ }^{8}$ Four patients scored "normal" for all health dimensions. The patient with aortic regurgitation also scored within $1 \mathrm{SD}$ of the mean for all areas except 

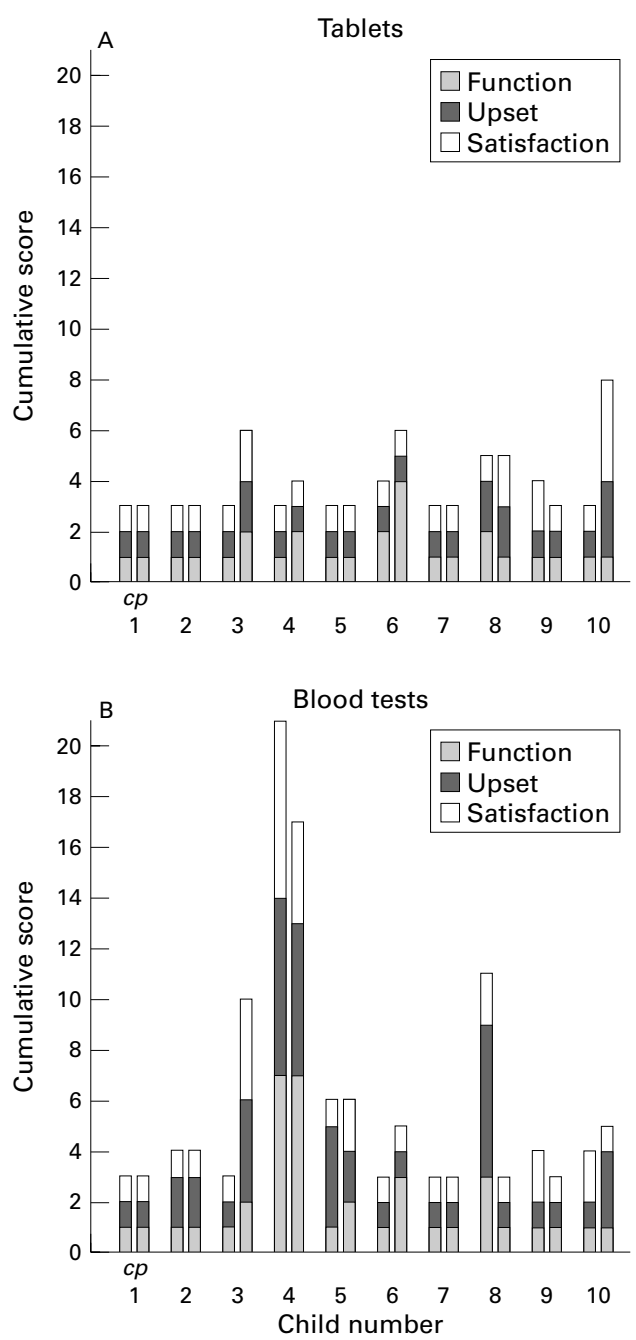

Figure 4 The effect of taking daily warfarin tablets $(A)$ and having blood tests $(B)$ on the child's quality of life as perceived by the child and parent. The level of function, upset, and satisfaction related to taking warfarin tablets and having blood tests were each scored on a scale between 1 and 7 and summed. The cumulative score ranged from 3 (no adverse effect on quality of life) to 21 (worst effect). Patient 4 suffers from needle phobia. c, child score; , parent score for the first child and each of the following children. See text and fig 1 for detailed description of questions and scoring system.

physical functioning. A further two patients, including the one with asthma, scored below the normal range for two of the eight health areas. Finally, two patients scored below the norm for nearly all areas of health.
On a scale of 0 (worst effect on QoL) to 100 (no adverse effect on QoL), taking daily warfarin tablets was rated between 80 and 100 by six patients and between 60 and 80 by two. The lowest score in this area (50) was by the only patient known to be non-compliant with warfarin treatment. The effect of having regular blood tests on QoL was between 80 and 100 by six patients and between 60 and 80 by three.

\section{Discussion}

Recent advances in operative techniques have made successful repair of the failing mitral valve possible for many patients, but sometimes valve replacement is the only solution. Unfortunately there is no ideal valve substitute, and mechanical prostheses, favoured because of their durability, require permanent anticoagulation. Taking daily warfarin tablets and having regular blood tests for control of anticoagulant treatment are thought to have a major impact on the quality of life of children after mitral valve replacement, but this had not been systematically investigated.

In our studies, none of the younger children or their parents perceived their child's health status as normal. The level of impairment was equal to or worse than that previously published for children with chronic disabilities. In contrast, most adolescents and young adults felt that their functioning in most areas was within normal limits. One can only speculate about the underlying reasons for this difference. It may be that the SF-36, as opposed to CQOL, was not sensitive enough to detect impaired health status following mitral valve replacement in the older patients. This seems unlikely, however, as it was shown in validation studies that the SF-36 was able to detect low levels of ill health. ${ }^{10}{ }^{11} \mathrm{~A}$ more likely reason may be that reduced health as a result of mitral valve replacement has a different impact on QoL in various age groups, possibly because the areas of life that are most important for general wellbeing change during a child's development. Normal exercise ability is important for the school age child to keep up with his peers. The more mature individual, however, usually has a wider choice of lifestyles and therefore physical health status may become less important for achieving a satisfactory QoL. It is also noteworthy that in spite of the reported impairment in health status, all but one patient

Table 2 Scores for the SF-36 questionnaire of nine patients ( $A$ to I) compared with a reference population matched for age and sex

\begin{tabular}{|c|c|c|c|}
\hline \multirow[b]{2}{*}{ Dimension } & \multicolumn{3}{|l|}{ Score } \\
\hline & $\begin{array}{l}\text { Within mean } \pm 1 S D \text { of } \\
\text { reference population }\end{array}$ & $\begin{array}{l}\text { Within mean } \pm 2 S D \text { of } \\
\text { reference population }\end{array}$ & $\begin{array}{l}\text { Outside mean } \pm 2 S D \text { of } \\
\text { reference population }\end{array}$ \\
\hline Physical function & A B C D E G & $\mathrm{H} \mathrm{I}$ & $\mathrm{F}$ \\
\hline Role limitation (physical) & A B D E G H I & $\mathrm{F}$ & $\mathrm{C}$ \\
\hline Role limitation (emotional) & A B D E G H I & $\mathrm{F}$ & $\mathrm{C}$ \\
\hline Social functioning & A B D E G H I & $\mathrm{C}$ & $\mathrm{F}$ \\
\hline Mental health & A B D G H I & E F & $\mathrm{C}$ \\
\hline Energy/vitality & A B D E G H I & $\mathrm{C}$ & $\mathrm{F}$ \\
\hline Pain & A B CDEFGH I & & \\
\hline General health perception & A B D G H & C E F I & \\
\hline
\end{tabular}

Normal score was defined as within 1 SD of the mean of the reference population.

Patient $\mathrm{H}$ suffers from aortic regurgitation and patient I from asthma. 
were able to participate in mainstream education or hold down a job.

Surprisingly, taking daily medication did not have an important effect on QoL in either age group, except in a 17 year old girl who had become non-compliant with her medication. Inspection of anticoagulation records revealed a stable level of anticoagulation in the majority of patients, suggesting adherence to the prescribed medication. Contrary to expectations, most young children and their parents reported that regular blood tests were well tolerated. In the older age group some degree of impaired QoL was related to either discomfort from the blood tests or disruption of daily life. The latter may be improved with the use of recently introduced commercial self test kits, reducing the need for patients to attend hospital for their blood sampling. ${ }^{12}$

It is increasingly important to measure the perception of health of patients to assess the benefits of treatment. This task is difficult because there is no uniform definition of health. Measurements in children are further complicated by the continuous development of the child and therefore in this patient population these measurements should be age specific. Although a large number of health status instruments have been developed over the years, a recent review found that many had insufficient psychometric properties. ${ }^{13}$ To avoid the above pitfalls we used age specific validated questionnaires. Because these questionnaires measured core functions of general health only, specific questions about the effects of permanent anticoagulation were added. In spite of the precautions taken with the selection of our instruments, it should be kept in mind that QoL measurements are still under development and that the outcomes of these types of studies should be viewed cautiously.

LIMITATIONS OF THE STUDY

A limitation of our study was that only a small number of patients were investigated. Mitral valve replacement in children is rare and therefore only a limited number of patients can be expected in a single centre. In addition, only selected patients were studied. Patients less than nine years old were not invited because we were unable to identify a satisfactory validated questionnaire, and patients resident outside the UK were excluded because of cultural bias of
QoL questionnaires. ${ }^{10} 13$ Finally, two patients with terminal heart failure and one patient with progressive deterioration of vision were not investigated for compassionate reasons, and there was a refusal and no response to the study invitation in a further two patients. One can only speculate on the effect of patient selection on the final outcome of this study.

\section{CONCLUSIONS}

The results of this study on QoL in children after mitral valve replacement indicate a moderate impairment in general health status for school age children and near normal QoL for the majority of adolescents and young adults. Permanent anticoagulation treatment was well tolerated in most patients. Currently available instruments for health status measurement need further development to allow better assessment of health related QoL in children.

We thank the children and their parents for participating in this study.

1 Almeida RS, Elliott MJ, Robinson PJ, et al. Surgery for congenital abnormalities of the mitral valve at the Hospital for genital abnormalities of the mitral valve at the Hospital for
Sick Children, London from 1969-1983. F Cardiovasc Surg Sick Children,

2 Zweng TN, Bluett MK, Mosca R, et al. Mitral valve replacement in the first 5 years of life. Ann Thorac Surg $1989 ; 47: 720-4$

3 Kadoba K, Jonas RA, Mayer JE, et al. Mitral valve replacement in the first year of life. $\mathcal{F}$ Thorac Cardiovasc Surg 1990; 100:762-8.

4 Harada Y, Imai Y, Kurisawa H, et al. Ten-year follow-up after valve replacement with the St Jude Medical prosthesis in children. F Thorac Cardiovasc Surg 1990;100:175-80.

5 Bradley SM, Sade RM, Crawford FA Jnr, et al. Anticoagulation in children with mechanical valve prostheses. An
Thorac Surg 1997;64:30-6.

6 van Doorn C, Yates R, Tsang V, et al. Mitral valve replacement in children: mortality, morbidity and haemodynamic status up to medium term follow up. Heart 2000;84:63642 .

7 Stevenson J. Health and illness in childhood. In: Sclare I, ed. Child psychology portfolio. Windsor, NFER-Nelson, 1997: Child psyc.

8 Jenkinson C, Layte R, Wright L, et al. The UK SF-36: an analysis and interpretation manual. Oxford: University of Oxford, Health Services Research Unit, Department of public health and primary care, 1996:1-65.

9 Graham P, Stevenson J, Flynn F. A new measure of healthrelated quality of life for children: preliminary findings. Psychol Health 1997;12:655-65.

10 Brazier JE, Harper R, Jones NM, et al. Validating the SF-36 health survey questionnaire: new outcome measure for primary care. BMF 1992;305:160-4

11 Garratt AM, Ruta DA, Abdalla MI, et al. The SF 36 health survey questionnaire: an outcome measure suitable for survey questionnaire: an outcome measure suitable
routine use within the NHS? BMF 1993;306:1440-4.

12 Hasenkam JM, Kimose HH, Knudson L, et al. Self management of oral anticoagulant therapy after heart valve ment of oral anticoagulant therapy after heart va

$13 \mathrm{Pal}$ DK. Quality of life assessment in children: a review of conceptual and methodological issues in multidimensional health status measurements. F Epidemiol Community Health 1996;50:391-6. 